\title{
Directive properties of active coated nano-particles
}

\author{
Samel Arslanagić ${ }^{*}$, and Richard W. Ziolkowski ${ }^{2}$ \\ ${ }^{1}$ Department of Electrical Engineering, Electromagnetic Systems, \\ Technical University of Denmark, Denmark \\ ${ }^{2}$ Department of Electrical and Computer Engineering, University of Arizona, USA \\ *corresponding author, E-mail: sar@elektro.dtu.dk
}

\begin{abstract}
The directivities of the fields radiated by a variety of cylindrical and spherical active coated nano-particles, which are excited by their respective sources of illumination at optical frequencies, are investigated. Particular attention is devoted to the influence of the source location and optical gain constant on the directivities. While significant variations in the directivities are realized in the cylindrical cases for different source locations within and slightly outside the nano-particles and values of the optical gain constant, the corresponding spherical cases exhibit negligible differences.
\end{abstract}

\section{Introduction}

The performance of electrically small antennas (ESAs) is constrained by certain conventional limitations. For instance, if not matched properly, they are very inefficient radiators of electromagnetic energy, due to their intrinsic non-resonant nature, with accompanying low directivities [1]. While a variety of traditional matching techniques exist, the recent advent in metamaterial (MTM) research has, among other things, fostered truly novel means of matching ESAs [2]. In particular, the use of double-negative (DNG), epsilon-negative (ENG), and/or mu-negative (MNG) MTMs was found to lead to interesting and highly resonant properties in sub-wavelength configurations when properly combined with ordinary double-positive (DPS) materials [2]-[5]. These resonances were exploited in [6] and [7], where efficient MTM-based ESAs were proposed, and in [8] where MTM-inspired electrically small near-field parasitic resonators were used as impedance transformers to realize matching of the overall antenna system to its source and to the wave impedance of the medium in which it radiates. While the above works focused on radio and microwave frequencies, similar highly resonant properties, for potential use in nano-sensing, -amplifying and -antenna applications, were reported for passive plasmonic-based configurations at optical frequencies, see e.g., [9], [10] and the works referenced therein, as well as active plasmonic-based configurations, see e.g., [11] and the works referenced therein, as well as [12] and [13]. In particular, interesting enhancements of directivity patterns for higher order mode sub-wavelength radiators made of DPS materials in conjunction with passive MTMs or plasmonic materials, were reported in [10], this providing an alternate route towards sub-wavelength radiators with high directivities.

The present work examines the directivity properties of nano-antennas consisting of active coated nano-particles (CNPs) of cylindrical and spherical shape excited by their respective sources of illumination at optical frequencies. Such nano-antennas could potentially be used for biosensing purposes. Particularly, they hold the potential of enhancing the emission in the far-field of targeted molecules. In this regard the directivity patterns would then reveal the maximum direction of that radiation. Conversely, these directivity results would provide a basis for choosing the directions from which most of the incoming optical fields could be converted into large localized electromagnetic fields, which would be needed for molecular sensing purposes.

For spherical CNPs, the source of excitation is an electric Hertzian dipole, which is taken to be normal as well as tangential to surfaces of the CNP, while for cylindrical CNPs, the source of excitation is an infinitely long magnetic line source. The CNPs emphasized inhere are all made of a silica nano-core and are layered with a concentric silver nano-shell; however, other variations in the materials of the CNP can be easily accounted for. The gain is introduced inside the dielectric part of the CNP, i.e., the nano-core, via the so-called canonical, constant frequency, gain model. This work constitutes an extension of [12] and [13] where detailed near-field studies have revealed super-resonant 
properties of the examined active CNPs despite their very small electrical sizes. Particular attention is devoted to the influence of the source location and the optical gain constant on the resulting directivity patterns. In the cylindrical case, it is shown how the directivity of a super-resonant CNP can be re-shaped by either changing the location of the line source or adjusting the value of the optical gain constant. As to the spherical CNPs, the resulting directivity patterns are shown to be unaffected by the presence of the CNP - even in its super-resonant state for which large amounts of the radiated power are extracted from the source - and they largely correspond to the pattern of an isolated dipole. Thus, as in the passive spherical case [10], the dipolar resonance excited inside the examined active spherical CNPs does not modify the directivity pattern of the exciting Hertzian dipole, but rather enhances its total radiated power.

This paper is organized as follows. Section 2 presents the configurations to be discussed, while the methods of their analysis and the associated analytical results are summarized in Section 3. The numerical results are presented in Section 4, and the entire work is concluded in Section 5. Throughout the work, the time factor $\exp (j \omega t)$, with $\omega$ being the angular frequency, and $t$ being the time, is assumed and suppressed.

\section{Configurations}

We consider both cylindrical (Figure 1a)) and spherical (Figure 1b)) CNPs.

In both cases, they consist of a dielectric nano-core (with permittivity $\varepsilon_{1}$, permeability $\mu_{1}$, and wave number $k_{1}$ ) covered concentrically with a plasmonic nano-shell (with permittivity $\varepsilon_{2}$, permeability $\mu_{2}$, and wave number $k_{2}$ ), and are immersed in free-space, which has the permittivity, $\varepsilon_{0}$, permeability, $\mu_{0}$, and thus the intrinsic impedance $\eta_{0}=\sqrt{\mu_{0} / \varepsilon_{0}}$ and wave number $k_{0}=\omega \sqrt{\varepsilon_{0} \mu_{0}}=2 \pi / \lambda$, where $\lambda$ denotes the free-space wavelength.

For the cylindrical CNP, the inner and outer radii of the nano-shell are denoted by $\rho_{1}$ and $\rho_{2}$, respectively. The CNP is illuminated by the field generated by an arbitrarily located infinitely long magnetic line source (MLS) possessing a constant magnetic current $I_{m}[\mathrm{~V} / \mathrm{m}]$. A circular cylindrical coordinate system, with the coordinates $(\rho, \phi, z)$ and an associated rectangular coordinate system $(x, y, z)$ are introduced such that their origins coincide with the cross-sectional center of the CNP. Furthermore, the entire CNP-MLS configuration is infinite in the z-direction, with the MLS being parallel to the axis of the cylinders. The coordinates of the observation point are $(\rho, \phi)$, and those of the MLS are $\left(\rho_{s}, \phi_{s}\right)$.

For the spherical CNP, the inner and outer radii of the nano-shell are denoted by $r_{1}$ and $r_{2}$, respectively. The CNP is illuminated by the field generated by an arbitrarily oriented and located electric Hertzian dipole (EHD) with the dipole moment $\vec{p}=\hat{p}_{s} p_{s}$, where $\hat{p}_{s}$ is its orientation and $p_{s}[\mathrm{Am}]$ is its complex amplitude. A spherical coordinate system $(r, \theta, \phi)$ and the associated rectangular coordinate system $(x, y, z)$ are introduced such that the origin of these coincide with the center of the CNP. The coordinates of the observation point are $(r, \theta, \phi)$, and those of the EHD are $\left(r_{s}, \theta_{s}, \phi_{s}\right)$.

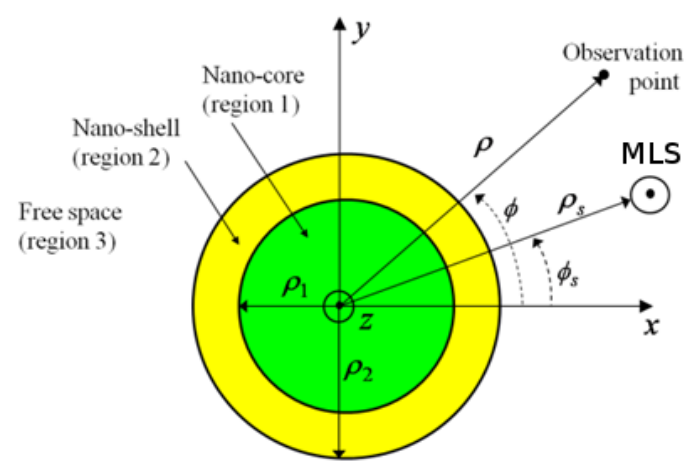

(a)

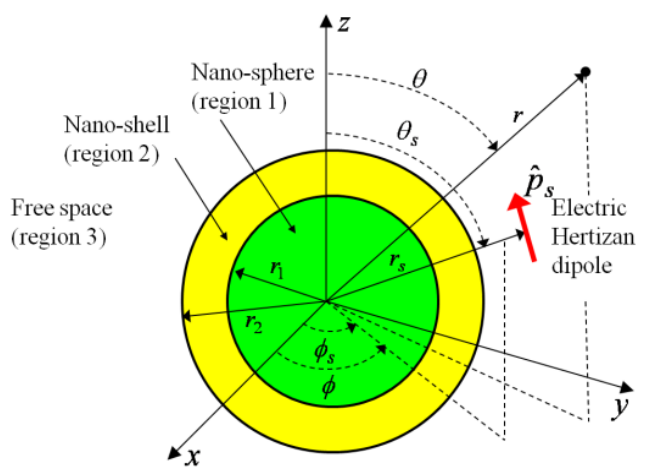

(b)

Figure 1: The cylindrical (a), and spherical (b) CNP configurations.

\section{Analytical results}

The analytical solutions for the problems shown in Figure 1 have been derived in [4] and [5], and are used here to study the directivity of these configurations.

For the cylindrical CNP configurations, the solution 
procedure is as follows. The field due to the MLS, which constitutes the known incident field, is expanded in terms of cylindrical wave functions. This is likewise the case with the unknown scattered fields due to the CNP in the three regions. The fields due to the CNP involve the unknown expansion coefficients $C_{i, n}$, where $i=1$ for the field in region $1, i=2$ and 3 for the field in region 2 , and $i=4$ for the field in region 3 , and where the symbol $n$ is the mode number ( $n=0$ in the expansion is the monopole mode, $n=1$ is the dipole mode, etc. for the other modes). The unknown expansion coefficients depend on the location of the MLS and are obtained by enforcing the boundary conditions at the two cylindrical interfaces, $\rho=\rho_{1}$ and $\rho=\rho_{2}$. With the exact field solutions at hand, the directivity, $D$, defined as the ratio of the radiation intensity to the total average power per unit angle, can be expressed as [4]

$$
D(\phi)=\frac{2 \cdot\left|\sum_{n=0}^{N_{\max }} \tau_{n} j^{n} \alpha_{n} \cos \left[n\left(\phi-\phi_{s}\right)\right]\right|^{2}}{\sum_{n=0}^{N_{\max }} \tau_{n}^{2}\left(3-\tau_{n}\right)\left|\alpha_{n}\right|^{2}},
$$

where $\alpha_{n}=C_{4, n}$ for the MLS inside the CNP, and $\alpha_{n}=J_{n}\left(k_{0} \rho_{s}\right)+C_{4, n}$ for the MLS outside the CNP. In these results, the function $J_{n}(\cdot)$ is the Bessel function of order $n, \tau_{n}$ is the Neumann number $\left(\tau_{n}=1\right.$ for the $n=0$ mode and $\tau_{n}=2$ otherwise), while $N_{\max }$ is the truncation limit in the implementation of the exact infinite summation chosen to ensure the convergence of the expansion in (1). The expression in (1) was used to study the directivity properties of sub-wavelength passive MTMbased particles in [4], and of super-resonant active CNPs in [13] for a variety of MLS locations.

For the spherical CNP configuration, the solution procedure is as follows. The field due to the EHD, which constitutes the known incident field, is expanded in terms of transverse magnetic (TM) and transverse electric (TE) spherical waves with the known expansion coefficients $a_{n m}^{(c)}$ (TM coefficients), and $b_{n m}^{(c)}$, (TE coefficients), where the index $c$ denotes the region in which the field is determined. The unknown scattered fields due to the CNP in the three regions are likewise expanded in terms of TM and TE spherical waves; these expansions involve the unknown TM and TE expansion coefficients denoted by $A_{i, n m}$ and $B_{i, n m}$, respectively, where $i=1$ for the field in region $1, i=2$ and 3 for the field in region 2 , and $i=4$ for the field in region 3 . These expansion coefficients depend on the EHD location and orientation, and are easily obtained by enforcing the boundary conditions on the two spherical interfaces, $r=r_{1}$ and $r=r_{2}$. The general field solutions have been obtained in [5] and are specialized here for the far-field observation points in order to derive an expression for the directivity of the configuration in Figure 2b). In particular, the directivity takes on the form,

$$
D(\theta, \phi)=\frac{2 \pi}{\eta_{0}} \frac{1}{P_{t}}\left(\left|F_{t, \theta}\right|^{2}+\left|F_{t, \phi}\right|^{2}\right),
$$

where

$$
\begin{aligned}
& F_{t, \theta}= \\
& \sum_{n=1}^{N_{\max }} \sum_{m=-n}^{n} j^{n-1} e^{j m \phi} \times \\
& {\left[\frac{1}{\omega \varepsilon_{0}} \alpha_{n m} \frac{d}{d \theta} P_{n}^{|m|}(\cos \theta)+\beta_{n m} \frac{1}{k_{0}} \frac{j m}{\sin \theta} P_{n}^{|m|}(\cos \theta)\right],}
\end{aligned}
$$

and

$$
\begin{aligned}
& F_{t, \phi}= \\
& \sum_{n=1}^{N_{\max }} \sum_{m=-n}^{n} j^{n-1} e^{j m \phi} \times \\
& {\left[\frac{1}{\omega \varepsilon_{0}} \alpha_{n m} \frac{j m}{\sin \theta} P_{n}^{|m|}(\cos \theta)-\beta_{n m} \frac{1}{k_{0}} \frac{d}{d \theta} P_{n}^{|m|}(\cos \theta)\right]}
\end{aligned}
$$

are, respectively, the $\theta$ - and $\phi$ - components of the total radiation vector, $\boldsymbol{F}_{t}(\theta, \phi)$, which is related to the total farfield term $\boldsymbol{E}_{t}(\theta, \phi)$ through the relation $\boldsymbol{E}_{t}(\theta, \varphi) \approx \boldsymbol{F}_{t}(\theta, \varphi) \exp \left(-j k_{0} r\right) / r$. Moreover, the quantity $P_{t}$ is the total radiated power, and is expressed as [5]

$P_{t}=\frac{\pi}{\omega k_{0}} \sum_{n=1}^{N_{\max }} \sum_{m=-n}^{n} 2 \frac{n(n+1)}{2 n+1} \frac{(n+|m|) !}{(n-|m|) !}\left[\frac{\left|\alpha_{n m}\right|^{2}}{\varepsilon_{0}}+\frac{\left|\beta_{n m}\right|^{2}}{\mu_{0}}\right]$.

In the above expressions, $\alpha_{n m}=A_{4, n m}$ and $\beta_{n m}=B_{4, n m}$, when the EHD is inside the two regions of the CNP, while $\alpha_{n m}=a_{n m}^{(4)}+A_{4, n m}$ and $\beta_{n m}=b_{n m}^{(4)}+B_{4, n m}$, when the EHD is outside the CNP [5]. Moreover, $P_{n}^{|m|}(\cdot)$ is the associated Legendre function of the first kind of degree $n$ and order $|m|$, while the symbol $N_{\max }$ is the truncation limit in the implementation of the exact infinite summation chosen to ensure the convergence of the expansion in (2). While the expression for the total radiated power in (4) was used in [5] and [12], [14] to conduct an analysis of sub-wavelength resonances in passive and active spherical CNPs, the directivity properties of such active particles on the basis of (2) have not been reported previously. 


\section{Numerical results and discussion}

\subsection{Background - sub-wavelength resonances in CNPs}

Previous results [11]-[14] have demonstrated that highly resonant sub-wavelength CNPs can be designed by including gain, e.g., inside their nano-cores. Such active configurations were found to lead to scattering crosssections that are orders of magnitude larger than the values predicted by their geometrical sizes, see e.g., [11] and the works referenced therein, as well as to the enormous radiated powers for localized excitation sources [12]-[14]. In those works, significant attention has been devoted to a $\mathrm{CNP}$ consisting of a silica $\left(\mathrm{SiO}_{2}\right)$ nano-core of radius $24 \mathrm{~nm}$, coated with a $6 \mathrm{~nm}$ thick silver (Ag) nano-shell for which the frequency and size-dependency of the permittivity was taken into account.

In the active CNP configuration, a canonical, constant frequency, gain model in which the gain is added to the lossless silica nano-core was considered. According to such a model, the silica nano-core permittivity is taken to be

$\varepsilon_{1}=\left(n^{2}-\kappa^{2}-2 j n \kappa\right) \varepsilon_{0}$,

where the parameter $n=\sqrt{2.05}$ is the refractive index of the silica nano-core in the frequency region of interest, and the parameter $\kappa$ determines the nature of the nano-core and thus of the CNP. In particular, it becomes lossless (and passive) for $\kappa=0$, it is lossy and passive for $\kappa>0$ (in which case $\kappa$ is referred to as the absorption coefficient [15] or optical loss constant [11]) and it is active for $\kappa<0$ (in which case it is referred to as the optical gain constant [11]). We note that both of the parameters, $n$ and $\kappa$, are contained in the expression for the wave number of the silica nano-core in the following manner:

$k_{1}=k_{0}(n-j \kappa)$.

For the active CNPs (consisting of a silica nano-core covered with a silver nano-shell) studied in [11]-[14], presently referred to simply as Ag-based CNPs, the so-called super-resonant states, with very large radiated powers, were identified for the cylindrical case for $\kappa=-0.175$ at the excitation wavelength of $\lambda=577.70 \mathrm{~nm}$, and for the spherical case for $\kappa=-0.245$ at the excitation wavelength of $\lambda=502.1 \mathrm{~nm}$. These super-resonances are illustrated in Figure 2 in terms of the normalized radiation resistance (NRR), i.e., the ratio of the total power radiated when the source and the CNP are present to the total power radiated by the source in the absence of the CNP, as a function of the source location, $x_{s}$. The sources are located along the positive $x$-axis for both the cylindrical and the spherical Ag- based CNPs. For the cylindrical CNP, the MLS possesses a current amplitude $I_{m}=1[\mathrm{~V} / \mathrm{m}]$, while both the $x$ - and $z$ oriented EHDs have a dipole moment $p_{s}=5 \cdot 10^{-9}$ [Am].

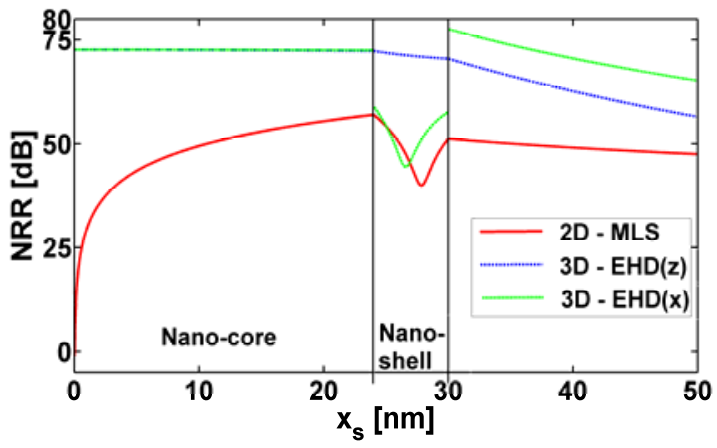

Figure 2: Normalized radiation resistance (NRR) as a function of the source location, $x_{s}$, along the positive $x$-axis for both the cylindrical and the spherical super-resonant Agbased CNPs. In the latter case, the EHD is taken to be both $x$ - and $z$-oriented. The silica nano-core and silver nano-shell regions are indicated in the figure.

Figure 2 displays notable differences between the cylindrical and spherical active CNP designs. All of the results in Figure 2 have been thoroughly accounted for via extensive near-field investigations for cylindrical [13] and spherical [12, 14] active Ag-based CNPs. We only summarize here the main points behind the distinct behaviors represented in Figure 2.

In the cylindrical case, very large variations in the NRR are observed. Specifically, no resonant phenomena exist for the MLS locations at or very close to the origin since the response for these locations is dominated by the monopolar mode ( $n=0)$, which is non-resonant $[4,13]$. As the MLS is moved closer to the nano-shell interfaces, very large NRR values result due to the excitation of a highly resonant dipolar mode ( $n=1)$, while the response decreases with the decreasing distance between the MLS (outside the CNP) and the CNP due to the slightly decreased coupling between the two. For the spherical case, there are practically no variations in the NRR with the varying source location inside the nano-core of the CNP. In the spherical case, a highly resonant dipolar mode is excited for all of the considered dipole locations $[5,12,14]$; this results in the observed very large NRR values. Apart from the differences between the two CNP geometries, Figure 2 also shows a few important differences in the spherical case between the two EHD orientations when they are located inside the nanoshell and outside the CNP [14]. When the $x$-oriented EHD is 
inside the nano-shell, the excitation of the resonant dipolar mode is weak and some of the field gets trapped within the nano-shell, and thus does not get radiated. The result is the noticeable reduction in the NRR values within the nanoshell region. On the other hand, when the $x$-oriented EHD is outside the CNP, the NRR surpasses the value of the $z$ oriented EHD due to a stronger excitation of the resonant dipolar mode. This stronger excitation results from the fact that the $x$-oriented EHD not only excites the resonant dipolar mode of the CNP, but it also drives directly the surface plasmon polaritons on the outer shell. Because the nanoshell is thin, these plasmonic waves extend into the core and in turn get amplified by the presence of the gain medium. Due to their decaying amplitude behavior away from the interface, the amplification is not large because the resulting coupling to the core is weak. Thus, the end result is a somewhat larger NRR value for this particular $x$-oriented EHD location relative to other locations and relative to the $z$ oriented EHD.

The directivity properties of these cylindrical and spherical Ag-based CNPs are thoroughly examined below. Their distinctive radiation pattern behaviors are emphasized.

\subsection{Cylindrical CNPs}

As noted above, the large variations in the NRR for the cylindrical Ag-based CNPs shown in Figure 2, associated with the different MLS locations, were thoroughly explained in [13]. Here, it is of great interest to explore the directivity behavior of these configurations.

\subsubsection{MLS location and optical gain constant influence}

Figure 3a) shows the directivity (1) for the super-resonant $(\kappa=-0.175 ; \lambda=577.70 \mathrm{~nm})$ Ag-based CNP for the indicated MLS locations along the positive $x$-axis within the nano-core. At this wavelength, the silver nano-shell permittivity is $\varepsilon_{2}=\varepsilon_{0}(-14.49-j 1.67)$. As can be observed, there are large variations in the resulting patterns. The directivity in the super-resonant state can be re-shaped from a perfectly monopolar pattern (attained at $x_{S}=0 \mathrm{~nm}$ ) to a perfectly symmetric dipolar pattern (attained at $x_{S}=5 \mathrm{~nm}$ ) simply by varying the MLS location. The former case agrees well with the absence of any resonant phenomena observed in Figure 2 when the MLS is at or very near the center of the CNP (NRR around $0 \mathrm{~dB}$ ). On the other hand, the latter case (NRR around $45 \mathrm{~dB}$ for $x_{S}=5 \mathrm{~nm}$ ) indicates a strong excitation of the dipolar mode, the latter being necessary for the rapidly increasing NRR values in Figure 2 for MLS locations further away from the CNP center. These far-field results are in line with our discussion in Section 4.1 and the near-field distributions reported for this Ag-based CNP configuration in [13]. For the perfectly symmetric dipolar pattern in Figure 3a), the maximum directivity of 2 is found along the $\phi=0^{\circ}$ and $\phi=180^{\circ}$ directions. However, larger values can be obtained for specific MLS locations (closer to the CNP center) for which the dominant mode is a mixture of the monopolar and dipolar modes, rather than the resonant dipolar mode alone. For instance, the result obtained for $x_{s}=0.1 \mathrm{~nm}$ and given in Figure 3b) illustrates this point.

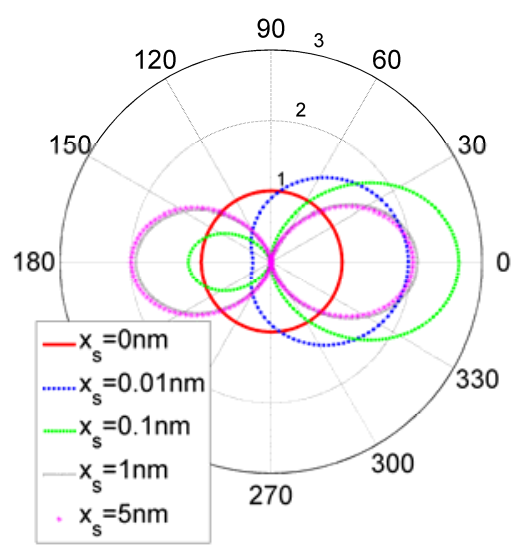

(a)

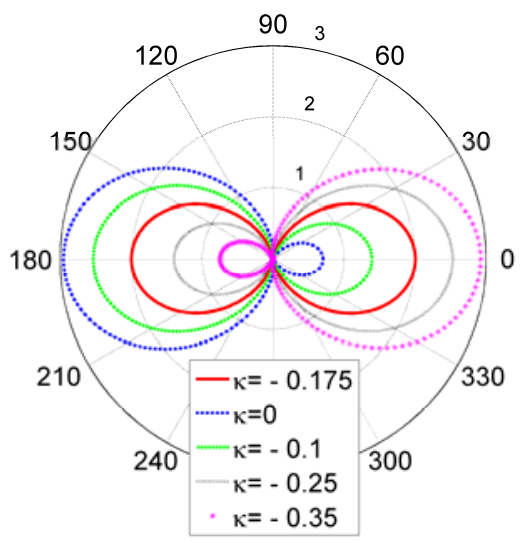

(b)

Figure 3: Directivity for the cylindrical Ag-based CNP excited by a MLS. The radius of the silica nano-core is 24 $\mathrm{nm}$ and the thickness of the silver nano-shell is $6 \mathrm{~nm}$. In (a), the CNP is super-resonant, and different MLS locations, $x_{s}$, inside the nano-core along the positive $x$-axis are considered. In (b), the MLS is located at $x_{s}=23 \mathrm{~nm}$ and different values of the optical gain constant $\kappa$ are considered.

Apart from the pattern re-shaping possibilities with varying the location of the MLS, it is interesting to note that 
an adjustment of the optical gain constant $\kappa$ for a given location of the source can likewise re-shape the overall directivity pattern. Figure $3 \mathrm{~b}$ ) shows the directivity for the Ag-based CNP when the MLS is located at $x_{s}=23 \mathrm{~nm}$ and the gain constant is varied. These results were obtained for the super-resonant wavelength $\lambda=577.70 \mathrm{~nm}$ and the corresponding silver nano-shell permittivity equal to $\varepsilon_{2}=\varepsilon_{0}(-14.49-j 1.67)$. As can be observed, the perfectly symmetric dipolar pattern attained in the superresonant case can be re-shaped by changing the gain constant, i.e., its main beam is along the $\phi=0^{\circ}$ or $\phi=180^{\circ}$ direction, depending on the value of $\kappa$. For the examined values of $\kappa$ which are larger than the superresonant value $(\kappa=-0.175)$, the main beam (which is approaching a maximum directivity of 3 ) points in the $\phi=180^{\circ}$ direction. In contrast, it points along the $\phi=0^{\circ}$ direction for values of $\kappa$ smaller than the superresonant value.

\subsubsection{Other remarks: MLS inside the silver nano-shell}

Aside from the interesting results for the cylindrical Agbased CNP reported in the previous sub-section, a few additional remarks are in order. As explained in [13] using the near-field distribution results, the main reason for the peak amplitude changes of the NRR in Figure 2 is the difference in the coupling strength as the MLS location is varied. This coupling strength determines the potency of the underlying resonant dipolar mode. As an example, the nearfield of the resonant dipolar mode is significantly stronger when the MLS is located near the nano-shell interface, e.g., at $x_{s}=23 \mathrm{~nm}$, than inside the nano-shell at $x_{s}=28.74 \mathrm{~nm}$, where the NRR experiences the minimum of its dip shown in Figure 2. However, once the MLS is sufficiently far away from the origin so that the perfectly symmetric dipolar pattern is obtained, as the one illustrated in Figure 3a) for $x_{s}$ $=5 \mathrm{~nm}$, the very same directivity pattern (of maximum directivity of 2) results also for other MLS locations inside the CNP, as well as outside of it at close distances. This is partly illustrated in Figure 4) which shows the directivity for three MLS locations inside the nano-shell of the superresonant Ag-based CNP: near the first interface $\left(x_{s}=25 \mathrm{~nm}\right)$, at the location of minimum NRR inside the nano-shell $\left(x_{s}=\right.$ $27.84 \mathrm{~nm})$, and near the second interface $\left(x_{s}=29 \mathrm{~nm}\right)$. In all cases, the same symmetric dipolar pattern is observed. Note that the explanation for the varying levels of the NRR found in Figure 2 within the nano-shell cannot be revealed by inspecting the directivity patterns (which, as shown in Figure 4, are identical), but rather the near-field distributions must be examined.

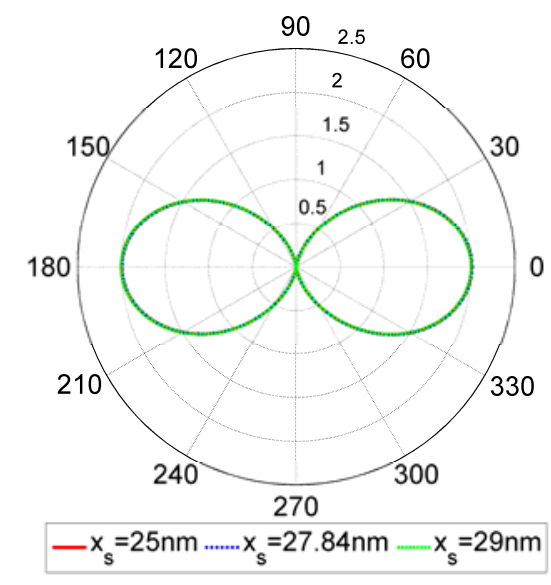

Figure 4: Directivity for the cylindrical super-resonant Agbased CNP excited by a MLS located at three different positions inside the nano-shell region of the particle. The radius of the silica nano-core is $24 \mathrm{~nm}$ and the thickness of the Ag nano-shell is $6 \mathrm{~nm}$.

\subsubsection{Comparison with MTM-based structures}

It is interesting to parallel the above results with those of the corresponding sub-wavelength resonant MTM-based structures at radio frequencies [5]. In [5], an electric line source excitation of double-negative (DNG) or mu-negative (MNG) coated passive structures was examined. As remarked there, those results also apply, by duality, to epsilon-negative (ENG) coated passive structures excited by a MLS. The maximum NRR levels in [5] for the resonant cylindrical MTM-based structures are well below (with maximum NRR around $23 \mathrm{~dB}$ ) those reported in Figure 2. Moreover, a complete symmetry in their dipolar directivity patterns was not obtained. In particular, for the source inside the ENG shell-region, asymmetric directivity patterns (which are each other's images with respect to the $y$-axis) result for MLS locations near the first and the second shell interface. This is in sharp contrast to the results of Figure 4 where identical and completely symmetric patterns are obtained. In addition, since the NRR of the MTM-based structures in [5] drops to $0 \mathrm{~dB}$ for a particular source location inside the shell-region, the pattern re-shaping also occurs for altering source locations in this region. Specifically, a perfect monopolar pattern is obtained for the minimum NRR location of the source inside the shell-region [5]. These findings for sub-wavelength resonant MTMbased ENG-coated passive structures appear in sharp contrast to the above gain-enhanced results for the active Ag-based CNPs. 


\subsection{Spherical CNPs}

Figure 2 reveals interesting differences between the $z$ - and $x$ oriented EHD excitations of the super-resonant Ag-based spherical CNP, which were detailed through near-field investigations in [14].

Figure 5 shows the directivity of the Ag-based CNP for varying values of the parameter $\kappa$ in the case of a $z$-oriented EHD excitation. The results are shown for the superresonant wavelength $\lambda=502.10 \mathrm{~nm}$, and the corresponding silver nano-shell permittivity $\varepsilon_{2}=\varepsilon_{0}(-9.81-j 1.16)$. The EHD has the dipole moment $p_{s}=5 \cdot 10^{-9}[\mathrm{Am}]$ and is located on the $x$-axis at $+12 \mathrm{~nm}$ from the origin. Specifically, the E-plane (xz-plane) pattern results are shown in Figure 5a) and the H-plane (xy-plane) pattern results are shown in Figure 5b). Despite the large levels of the NRR, the directivity of the super-resonant state, which is clearly dipolar, is not enhanced relative to that of an isolated $z$ oriented EHD. In particular, because it radiates as an electrically small dipole, its value remains around 1.5. Unlike the cylindrical case, there is a complete symmetry in the CNP and, consequently, no preferred axis. Moreover, the investigated values of $\kappa$ are found, again because of the symmetry, to have only a negligible effect on the directivity. The directivity of the super-resonant Ag-based CNP is shown in Figure 5c) as a function of $\theta \in\left(0^{\circ}, 180^{\circ}\right)$ and $\phi \in\left(0^{\circ}, 360^{\circ}\right)$, clearly supporting the above observations.

For the $x$-oriented EHD excitation of the super-resonant Ag-based CNP, Figure 6 shows the resulting directivity. The E-plane (xz-plane) pattern is shown in Figure 6a); the H-plane (yz-plane) pattern is shown in Figure 6b). The directivity as a function of $\theta \in\left(0^{\circ}, 180^{\circ}\right)$ and $\phi \in\left(0^{\circ}, 360^{\circ}\right)$ is shown in Figure 6c). Except from the flipping of the E-plane pattern by $90^{\circ}$ relative to the $z$ oriented EHD case in Figure 5a), the same over-all conclusions apply as with the z-oriented EHD case, i.e., the directivity in the E-plane is not enhanced by the presence of the super-resonant Ag-based CNP and its maximum value remains around 1.5, while in the H-plane (xy-plane), its pattern is azimuthally symmetric. This behavior is again expected because of the spherical symmetry of the CNP. Thus, as in the passive spherical case [10], the dipolar resonance excited inside and outside nearby a superresonant, active, electrically small spherical CNP does not modify the directivity pattern of the exciting Hertzian dipole, but rather reinforces it. Again note that the reason for the varying NRR levels observed for the $x$-oriented EHD in Figure 2 cannot be revealed through the directivity pattern alone, but rather require studying the near-field distributions.

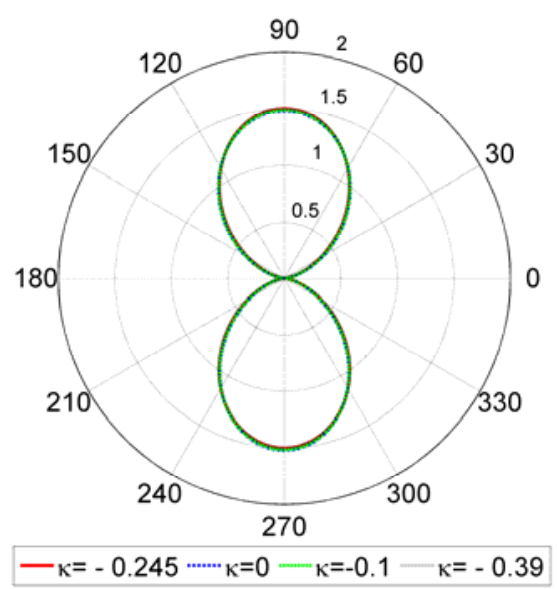

(a)

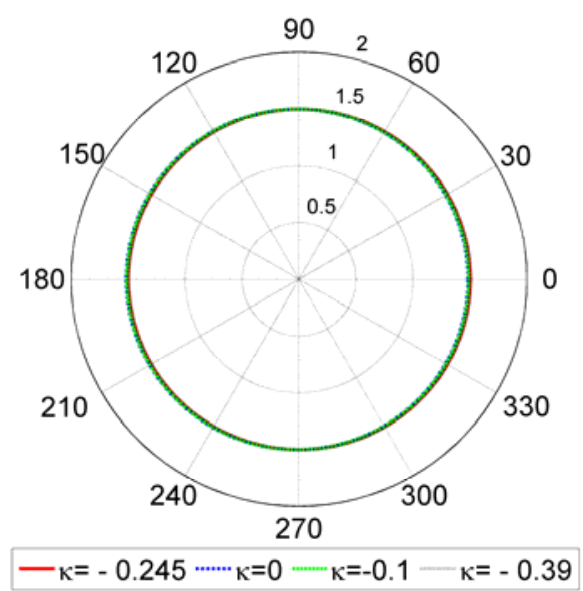

(b)

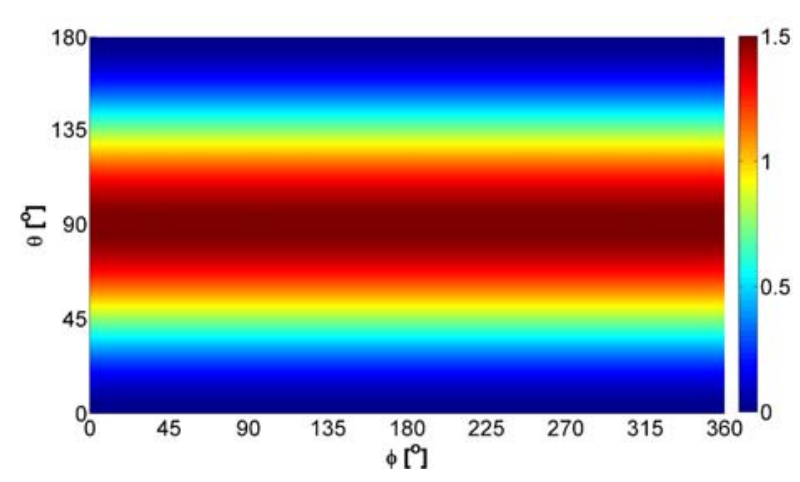

(c)

Figure 5: Directivity for the spherical Ag-based CNP for a zoriented EHD located along the $x$-axis at $+12 \mathrm{~nm}$ for different values of the parameter $\kappa$. The radius of the silica nano-core is $24 \mathrm{~nm}$ and the thickness of the silver nano-shell is $6 \mathrm{~nm}$. The E-plane ( $x z$-plane) pattern results are shown in (a), and the H-plane ( $x y$-plane) pattern results are shown in (b). In the case of the super-resonant Ag-based CNP, the directivity is shown in (c) for all values of $\theta$ and $\phi$. 


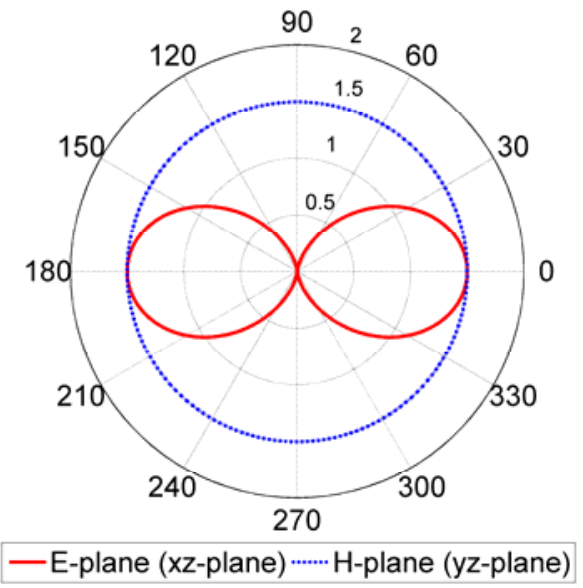

(a)

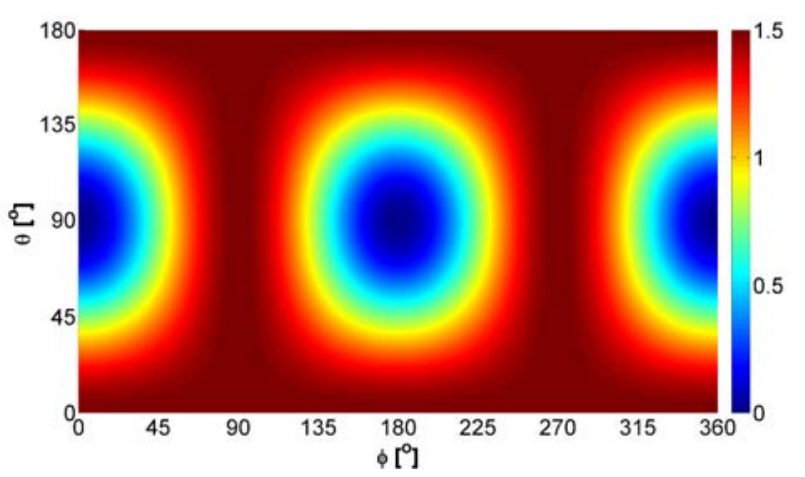

(b)

Figure 6: Directivity for the spherical super-resonant Agbased CNP for a $x$-oriented EHD located along the $x$-axis at $12 \mathrm{~nm}$. The radius of the silica nano-core is $24 \mathrm{~nm}$ and the thickness of the silver nano-shell is $6 \mathrm{~nm}$. The E-plane ( $x z-$ plane) and H-plane (yz-plane) results are shown in (a), while the results for all values of $\theta$ and $\phi$ are shown in (b).

\section{Conclusions}

This work examined the directivity properties of nanoantennas made of active CNPs of cylindrical and spherical shapes for potential use in bio-sensing applications. The CNPs were excited by a magnetic line source (MLS), in the cylindrical case, and an electric Hertzian dipole (EHD), in the spherical case. The nano-core of the particle consisted of silica, whereas silver was used for the nano-shell material. A canonical, constant frequency, gain model was incorporated inside the dielectric part of the particle. In our studies, particular attention was devoted to the influence of the source location and the optical gain constant on the resulting directivity patterns.

For cylindrical active CNPs, significant variation in the directivity was reported when either the MLS location or the value of the optical gain constant was varied. Specifically, pattern re-shaping from a perfectly monopolar form to a perfectly dipolar pattern (with maximum directivity of 2) was demonstrated for super-resonant CNPs upon the variation of the MLS location - the re-shaping being owed to the coupling strength with the varying MLS location which determines the potency of the underlying resonant dipolar mode. Moreover, the perfectly symmetric dipolar patterns of the super-resonant CNPs were re-shaped by proper adjustments of the optical gain constant; their main beams pointed in either the $\phi=0^{\circ}$ or $\phi=180^{\circ}$ directions, with the corresponding directivities of almost 3 . Moreover, it was found that, once the MLS location for a super-resonant CNP is sufficiently away from its center, the symmetric dipolar mode is excited, and it dominates the behavior of the directivity pattern. The pattern was found to remain unaltered for MLS locations inside the nano-core even at locations further away from the CNP center, as well as for those inside the nano-shell, and outside the CNP at close distances to it. These results are in sharp contrast to those reported for corresponding passive MTM-based configurations at radio frequencies.

The directivity patterns for the active spherical CNPs were shown to be unaffected by variations in the parameters specifying their configurations - even in their super-resonant state for which large amounts of the radiated power are generated by the source. In particular, it was demonstrated that they largely correspond to the pattern of an isolated electrically small dipole, i.e., their maximum value remained around 1.5. Unlike the cylindrical case, there is a complete symmetry in the CNP and, consequently, no preferred axis. Thus, as in the passive spherical case treated in [10], the dipolar resonance excited inside the active spherical CNPs does not modify the directivity pattern of the exciting Hertzian dipole. The potential excitation of higher order modes in other configurations of the active CNPs will be considered in a future report.

\section{References}

[1] R. E. Collin, Antennas and Radiowave Propagation, McGraw-Hill, Int. Ed., 1985.

[2] N. Engheta, and R. W. Ziolkowski, MetamaterialsPhysics and Engineering Applications, John Wiley \& Sons, New York, 2006.

[3] A. Alú, and N. Engheta, "Polarizabilities and effective parameters for collections of spherical nano-particles fromed by pairs of concentric double-negative (DNG), single-negative (SNG) and/or double-positive (DPS) metamaterial layers," J. Appl. Phys., Vol. 97, p. 094310, May 2005.

[4] S. Arslanagić, R. W. Ziolkowski, and O. Breinbjerg, "Analytical and numerical investigation of the radiation from concentric metamaterial spheres excited by an electric Hertzian dipole," Radio Sci., Vol. 42, 2007, doi:10.1029/2007RS003663.

[5] S. Arslanagić, R. W. Ziolkowski, and O. Breinbjerg, "Analytical and numerical investigation of the radiation and scattering from concentric metamaterial cylinders excited by an electric line source," Radio Sci., Vol. 42, 2007, doi:10.1029/2007RS003644. 
[6] H. R. Stuart, and A. Pidwerbetsky, "Electrically small antenna elements using negative permittivity radiators," IEEE Trans. Antennas Propag., Vol. 54, No.6, pp. 1644-1653, June, 2006.

[7] R. W. Ziolkowski, and A. Erentok "Metamaterial-based efficient electrically small antennas," IEEE Trans. Antennas Propag., Vol. 54, No. 7, pp.2113-2130, July 2006.

[8] A. Erentok, and R.W. Ziolkowski, "Metamaterialinspired efficient electrically small antennas," IEEE Ant. Propag., Vol. 56, No. 3, 691-707, 2008.

[9] S. J. Oldenburg, G. D. Hale, J. B. Jackson, and N. J. Halas, "Light scattering from dipole and quadrupole nanoshell antennas," Appl. Phys. Lett., Vol. 75, No. 8, pp. 1063-1065, Aug. 1999.

[10]A. Alú, and N. Engheta, "Enhanced directivity from sub-wavelength infrared/optical nano-antennas loaded with plasmonic materials or metamaterials," IEEE Trans. Ant. Propag., Vol. 55, No. 11, 3027-3039, 2007.

[11] J. A. Gordon, and R. W. Ziolkowski, "The design and simulated performance of a coated nano-particle laser," Opt. Express, Vol. 15, No. 5, 2622-2651, 2007.

[12] S. Arslanagić, and R. W. Ziolkowski, “Active coated nano-particle excited by an arbitrarily located electric Hertzian dipole - resonance and transparency effects," J. Opt., Vol. 12, 024014, 2010.

[13] S. Arslanagić, Y. Liu, R. Malureanu, and R. W. Ziolkowski, "Impact of the excitation source and plasmonic material on cylindrical active coated nanoparticles," Sensors, Vol. 11, 9109-9120, 2011.

[14] R. W. Ziolkowski, S. Arslanagić, and J. Geng, "Where high-frequency engineering advances optics: active nanoparticles as nanoantennas," to appear in Optical Nanoantennas, Eds. A. Alú and M. Agio, Cambridge University Press, London, 2012.

[15] L. D. Landau and E. M. Lifshitz, Electrodynamics of Continuous Media, Oxford: Pergamon, 1960. 